\title{
Shared Response Preparation for Pursuit and Saccadic Eye Movements
}

\author{
Dorion Liston ${ }^{1,2}$ and Richard J. Krauzlis ${ }^{1}$ \\ ${ }^{1}$ Systems Neurobiology Laboratory, Salk Institute for Biological Studies, La Jolla, California 92037, and ${ }^{2}$ Graduate Program in Neurosciences, University of \\ California San Diego, San Diego, California 92093
}

Tracking a single target in the visual world requires coordination between pursuit and saccadic eye movements. The constraints imposed on pursuit and saccade decisions by visual processing and response preparation are difficult to compare because latency differences between the two movements provide different amounts of visual sampling time. The present study compares pursuit and saccade decisions when visual processing was directly manipulated. Human observers were asked to select between two stationary stimuli presented simultaneously at two different locations based on which had the higher contrast. The stimuli were presented for a brief, variable interval and then occluded by masks. Because the masks moved horizontally and were offset vertically, subjects were obliged to make both pursuit and saccadic eye movements to track the mask covering the target stimulus. For each of the exposure durations, we constructed oculometric curves for pursuit and saccades. We found that both systems had similar oculometric thresholds and response biases. The initial pursuit decisions differed from the subsequent saccade decisions on 1-13\% of the trials but were the same more often than predicted by independent mechanisms. Moreover, pursuit reversed direction on discordant trials, so that the pursuit decision always matched that of the saccade by the time the saccade was started. These results support the view that, in addition to overlap in early visual areas and the final motor pathways, the pursuit and saccadic systems share processing at the level of response preparation. This shared processing may help ensure the coordination of pursuit and saccadic eye movements in selecting a single target.

Key words: pursuit; saccade; human; target selection; oculometric; attention; choice behavior; discrimination

\section{Introduction}

Primates generate two types of voluntary eye movements while exploring their visual environment: pursuit and saccades. Pursuit uses motion information about the selected object to match eye velocity to target velocity, thereby preventing the blurred vision that would otherwise be caused by the motion of the object's image across the retina (Lisberger et al., 1987; Krauzlis and Stone, 1999). Saccades use spatial information about the selected object to align the eyes with the target, thereby placing the image of the object near the fovea (Sparks and Mays, 1990; Schall and Thompson, 1999). Because the two types of movements almost always target the same goal, their programming necessarily involves some form of coordination.

Behavioral evidence of this coordination has been provided by examining how changes in experimental conditions affect pursuit and saccade latencies. The introduction of a temporal gap between fixation point offset and the appearance of a visual target decreases the latencies of both pursuit (Merrison and Carpenter, 1995; Knox, 1996; Krauzlis and Miles, 1996) and saccades

Received July 15, 2003; revised 0ct. 1, 2003; accepted 0ct. 6, 2003.

This research was supported by NASA NCC2-1024, National Eye Institute Grant EY 12212, and the Chapman Charitable Trust. D. Liston was supported by Merck and a graduate research fellowship from National Science Foundation. We are grateful to Geoff Boynton and Lee Stone for helpful fitting code, Brent Beutter for statistical advice, and Garth Fowler and Leanne Chukoskie for helpful comments on this manuscript.

Correspondence should be addressed to Richard J. Krauzlis, Salk Institute for Biological Studies, 10010 North Torrey Pines Road, La Jolla, CA 92037. E-mail: rich@salk.edu.

Copyright $\odot 2003$ Society for Neuroscience $\quad$ 0270-6474/03/2311305-10\$15.00/0
(Saslow, 1967; Fischer and Boch, 1983; Fischer and Weber, 1993). These decreases show a similar dependence on the duration of the temporal gap (Krauzlis and Miles, 1996), suggesting that there are common inputs for triggering the two types of eye movements (Krauzlis and Miles, 1996). The presence of a distractor in the hemifield opposite a visual target increases the latencies of pursuit and saccades (Ferrera and Lisberger, 1995; Walker et al., 1997; Krauzlis et al., 1999; Knox, 2001; Knox and Bekkour, 2002); the similarity of the effects on pursuit and saccades provides further evidence that the triggering of the two movements involves shared signals, perhaps related to visual attention or the control of ocular fixation.

Although suggestive, effects on latency provide ambiguous information about the processing leading up to movement onset, because reaction times depend on interactions between a diverse set of factors, including sensory processing, attentional modulation, decision criteria, and motor constraints. An alternative approach for teasing these factors apart is to treat eye movement responses as though they were perceptual reports and use techniques from psychophysics to quantify the performance of the two systems (Kowler and McKee, 1987; Beutter and Stone, 1998). For example, how well do pursuit and saccades discriminate the target? Do they exhibit biases in their decision criteria? How do these properties change over time? These questions have been addressed only tangentially in previous studies. Error rates for saccades systematically increase as targets in a search array become less distinguishable from the distractors (Eckstein et al., 


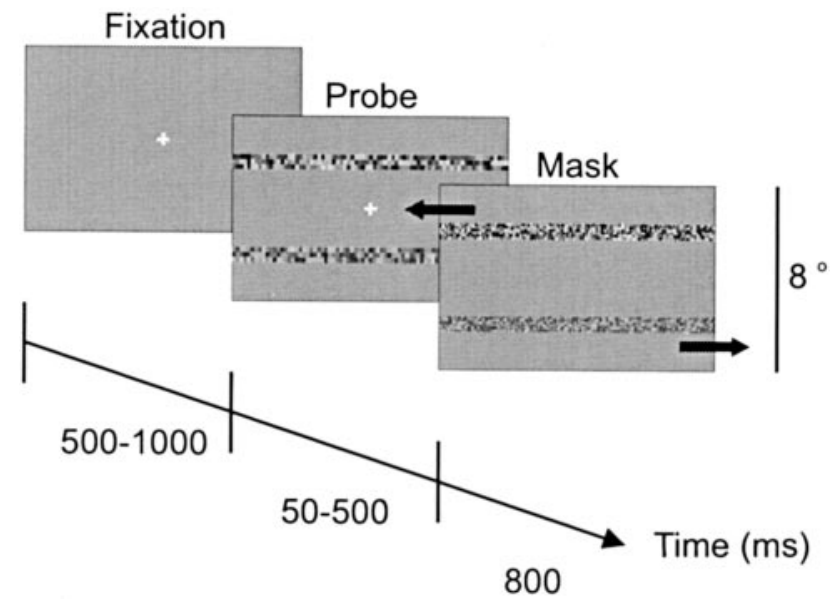

Figure 1. Schematic diagram of one trial. The sequence of the three screen images depicts the three intervals that occurred over the course of a trial. Each screen image represents the central portion $\left(11.7^{\circ}\right.$ hor $\times 8^{\circ}$ ver) of the video monitor. The large black arrows in the mask interval signify the opposed horizontal motion of the masks and did not appear on the monitor.

2001), but pursuit error rates have been measured only in cases in which the target and distractor are either indistinguishable (Lisberger and Ferrera, 1997) or easily identified (Ferrera and Lisberger, 1995; Krauzlis et al., 1999).

In the present study, we used a contrast discrimination task to compare the performance of pursuit and saccades. Because pursuit and saccades occur with different latencies, we presented the discrimination stimuli for only a brief interval and then replaced them with masks, so that the period of visual processing relevant for target selection was the same for both systems. We report that pursuit choices initially differed from those of the later saccade on between 1 and $13 \%$ of the trials, but that pursuit responses changed over time so that the two systems were in agreement by saccade onset. The initial discordance and its subsequent resolution appear to be caused primarily by a time-varying bias for spatial location.

\section{Materials and Methods}

Subjects. Five adult subjects participated in this experiment: the two authors (subjects D and R) and three naive observers. Each subject participated in a total of 13 sessions, the first 3 of which were training sessions. The data used in this study were collected in the last 10 sessions, each lasting $\sim 45 \mathrm{~min}$.

Stimuli and paradigm. Stimuli were presented on a video monitor (Eizo FX-E7) with a refresh rate of $120 \mathrm{~Hz}$ positioned $41 \mathrm{~cm}$ from the subjects. The display subtended an area $29.3^{\circ}$ vertical (ver) by $45^{\circ}$ horizontal (hor). The stimuli were generated using VisionWorks software (Swift et al., 1997). The temporal sequence of the stimuli presented during a single experimental trial is illustrated in Figure 1. During the initial "fixation" interval, subjects fixated a central $0.5^{\circ}$ cross. After a random 500-1000 msec interval, two stationary probe "noise" strips $\left(0.7^{\circ}\right.$ ver $\times$ $40^{\circ}$ hor $)$ were presented above and below the fixation cross $\left( \pm 2^{\circ}\right)$. These strips were composed of small $\left(0.24^{\circ}\right.$ ver $\times 0.20^{\circ}$ hor $)$ rectangles with luminances drawn from a uniform distribution with mean equal to the background luminance $\left(33 \mathrm{~cd} / \mathrm{m}^{2}\right)$. We used a look-up table to correct for the gamma of the monitor. Contrast is given by the equation (DeValois and DeValois, 1991): Contrast $=(\operatorname{Max}-\operatorname{Min}) /(\operatorname{Max}+\operatorname{Min}) \times$ $100 \%$, in which Max and Min are the maximum and minimum values in this luminance distribution, respectively. One probe, which was the target of the tracking task, was assigned a contrast that varied from 50 to $75 \%$ in steps of $5 \%$. The second probe acted as a distractor and had contrast held constant at $50 \%$. The duration of the "probe" interval was varied from 50 to $500 \mathrm{msec}$, and we will refer to this interval as the exposure duration. Because of video refresh, the actual display duration of the probe interval was an integer multiple of the time required to redraw the screen once, so the reported values of exposure duration are accurate to within $8.3 \mathrm{msec}$. After the probe interval, masks replaced the probe strips and the fixation point disappeared. During the "mask" interval, subjects viewed masks that were the same size as the probes and appeared in the same locations, but the rectangles had a higher spatial frequency $\left(0.12^{\circ}\right.$ ver $\times 0.10^{\circ}$ hor $)$ and the masks moved horizontally in opposite directions at $14.2^{\circ} \mathrm{sec}$. The motion of the masks was randomly assigned on each trial and counterbalanced across trials. Observers were asked to make an eye movement to and follow the mask at the location of the higher-contrast probe stimulus.

In our experiment, two types of visual processing were necessary to perform the task: visual processing necessary to select the target and visual processing necessary to drive the movement. We designed our experiment to control for the visual processing necessary for target selection; we limited the duration of the probe interval to directly manipulate the period of visual processing necessary for selection of the highercontrast probe. After this visual processing had transpired, the subjects could then begin visual processing necessary to drive the movement. The saccadic system had an advantage in this stage of processing because the location of the target mask was known before it appeared, whereas its motion was not. The oculometric methods described later quantify how well pursuit and saccades perform in discriminating target from distractor using information obtained during the probe interval, namely, visual processing necessary for target selection.

We used extended horizontal strips as pursuit stimuli, rather than the spot stimuli that are more typical, so that the retinal location of the visual stimulus was independent of pursuit latency. For typical stimulus configurations with a moving spot, longer pursuit latencies will result in the spot being at a more eccentric retinal position at the time of pursuit initiation. This results in (1) lower acuity visual stimuli for long latency pursuit and (2) changes in the need for corrective saccades as pursuit latency changes. In particular, long latency pursuit of moving spots is more likely to begin with a corrective saccade, and therefore be excluded from analysis, introducing a possible sampling bias. The horizontal strips avoided these problems by keeping the eccentricity of the stimulus fixed at $2^{\circ}$ regardless of latency. With these stimuli, we obtained at least 30 msec of saccade-free pursuit on $83 \%$ of the trials, with latencies ranging from $\sim 125$ to $200 \mathrm{msec}$.

The contrast of each mask was selected independently at random to be between 25 and 100\% contrast, at increments of 5\%. The difference in contrast between the target mask and the distractor mask was always assigned to be greater than the difference in contrast between the target probe and the distractor probe. This constraint was used to defeat a potential strategy whereby subjects could reliably identify the target probe strip by looking for the larger of the two relative contrast changes between the probe strips and the masks. Thus, the difference in contrast between the probe noise strips provided the only information as to the identity of the target.

We recorded the subjects' eye movements throughout the trial. We confirmed that subjects maintained fixation during the fixation interval and the probe interval by analysis of the eye-movement traces from each trial. If a subject made a saccade of $\geq 0.5^{\circ}$ during the fixation interval, the trial was discarded. Otherwise, saccades around the fixation point with smaller amplitudes were ignored and the trial was retained.

Data collection. The experiment was executed on a personal computer running the Tempo software package (Reflective Computing, St. Louis, $\mathrm{MO})$. Tempo controlled the timing of the stimuli, acquired the data, and saved it to disk. An additional personal computer equipped with a highspeed graphics card (VSG2/3; Cambridge Research Systems, Kent, UK) and VisionWorks software (Vision Research Graphics, Durham, NH) acted as a server to present the visual stimuli under the control of the Tempo machine. The visual display computer returned trigger signals to the Tempo machine as each stimulus was displayed, allowing us to synchronize data collection with stimulus presentation with $1 \mathrm{msec}$ resolution.

Eye movements were measured with an infrared video-based eye tracker (RK-726; ISCAN Inc., Burlington, MA). The eye tracker reported the horizontal and vertical positions of the pupil with 12-bit resolution 
using an algorithm that computes the centroid of the pupil at $240 \mathrm{~Hz}$. During the experimental sessions, subjects used a bite bar to minimize measurement errors caused by head movements. We calibrated the output from the eye tracker by recording the raw digital values as subjects fixated a set of known locations three times in a pseudorandom sequence. We used the mean values during $500 \mathrm{msec}$ intervals at each location to generate a smooth function (using cubic spline interpolation) for converting the raw eye tracker values to horizontal and vertical eye position. The measurement noise caused by the eye tracker was $\sim 0.05^{\circ}$ (the SDs of the measurements from these $500 \mathrm{msec}$ intervals), and the accuracy of the eye tracker measurements was $\sim 0.10^{\circ}$ (the SD of the mean values over these $500 \mathrm{msec}$ intervals obtained with repeated fixations). All eyemovement data and signals related to onset of stimuli were stored on the hard disk of the Tempo machine during the experiment and later transferred to a freeBSD system (Berkeley Software Distribution) for off-line analysis.

An interactive analysis program was used to display and make measurements from the data. Signals encoding eye velocity were obtained by applying a finite impulse response (FIR) filter $(-3 \mathrm{~dB}$ at $54 \mathrm{~Hz})$ to the calibrated eye position signals. Signals encoding eye acceleration were then obtained by applying the same FIR filter to the signals encoding velocity. We detected saccades by applying a set of amplitude criteria to radial eye velocity and radial eye acceleration signals, similar to what has been described previously (Krauzlis and Miles, 1996). We also visually inspected and verified each of the saccade measurements. For the analysis of pursuit data, we excised the interval containing the saccade from the horizontal eye velocity traces, so we did not confound pursuit measurements with the horizontal components of oblique saccades. Saccades in our experiment were primarily vertical but tended to deviate toward the direction of motion, on average resulting in a horizontal position component of $\sim 1.42^{\circ}$ (vertical, $\sim 1.83^{\circ}$ ) and an average saccade vector rotated $31^{\circ}$ away from exactly vertical.

The onset of pursuit was estimated from eye velocity traces using a variation of an algorithm described previously (Krauzlis and Miles, 1996). In this previous technique, the variance associated with a "baseline" interval was used to detect the beginning of a "response" interval. A linear regression of the response interval as a function of time was used to determine when the response intersected the baseline; this point in time was defined as the latency of pursuit. The extrapolation used in this method makes the latency estimates sensitive to noise in the response interval, and this problem is worse with the somewhat higher noise associated with video-based methods of eye movement recording. We therefore constrained the response interval to immediately follow, and be continuous with, the baseline interval, forming a "hinge" (Adler et al., 2002). The baseline and response intervals both were assigned durations of $100 \mathrm{msec}$, and we tested possible hinge placements ranging from \pm 30 $\mathrm{msec}$, in increments of $1 \mathrm{msec}$, from an initial subjective estimate of pursuit latency. For each of these hinge placements, the slope of the response interval was determined by linear regression, and we measured the mean squared error between the data and the model (baseline plus response intervals). The hinge placement that minimized the mean square error was defined as the latency of pursuit, and the slope during the $100 \mathrm{msec}$ response interval was recorded as pursuit acceleration.

For each trial, we measured pursuit latency, pursuit acceleration, saccade latency and offset, and the horizontal and vertical components of saccade amplitude. The initial pursuit decision (rightward or leftward) was defined to be the sign of pursuit acceleration in the "open loop" interval, corresponding to the $100 \mathrm{msec}$ response interval of the hinge model. The initial saccade decision (upward or downward) was defined to be the sign of the vertical component of saccade amplitude. We determined that we could reliably measure the initiation of pursuit without interference from the saccade when pursuit began $\geq 30 \mathrm{msec}$ before the saccade. In all of our analyses, we have included only trials in which pursuit onset occurred $\geq 30 \mathrm{msec}$ before the saccade.

For trials on which pursuit began in one direction and then reversed, we made measurements for both the initial decisions and the reversal. Because pursuit reversal did not occur after a fixed period of unchanging eye velocity (i.e., after a period of fixation, after steady-state tracking), the hinge model method of determining when pursuit began was unsuitable

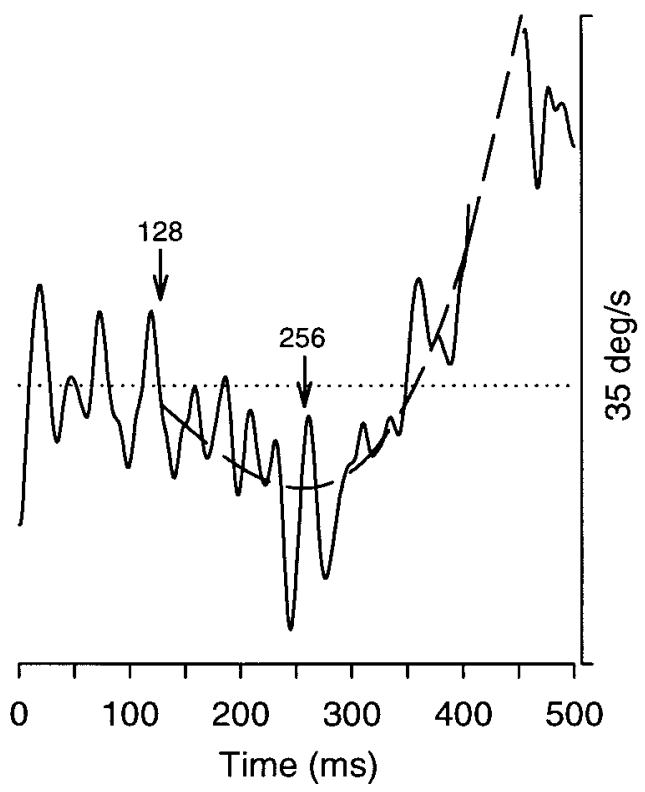

Figure 2. Measurement of pursuit turnarounds. The solid line plots horizontal eye velocity as a function of time with respect to mask onset. The dashed line plots a third-order polynomial fit to the horizontal eye velocity trace. Zero velocity is shown as a dotted line. We determined the time point at which pursuit turned around by finding the roots of the polynomial fit. Pursuit initially follows the distractor mask (latency marked at $128 \mathrm{msec}$ ) and then turns around to follow the target mask at $256 \mathrm{msec}$. The saccade, which occurred at $406 \mathrm{msec}$, has been excised from the horizontal velocity trace.

for measuring pursuit turnarounds. Therefore, we fit the horizontal eye velocity with a polynomial to determine the point in time when pursuit reversed direction (Fig. 2). This figure plots measured eye velocity as a function of time as a solid line and the polynomial fit to that velocity as a dashed line. The interval over which we fit the velocity trace began at the time of initial pursuit initiation, marked with an arrow at $128 \mathrm{msec}$. The interval continued until pursuit reached $75 \%$ of the velocity of the final pursuit target. In the example case, this occurred at the first point in time after the saccade, which has been excised, as in all horizontal eye velocity traces. The best-fit third-order polynomial over this interval gave us an objective estimate of the point in time when pursuit switched. We defined this point to be a root of the third-order polynomial fit, a point at which eye acceleration goes to zero, marked in Figure 2 at $256 \mathrm{msec}$. The other root was either not in the measurement interval or occurred at pursuit initiation, either of which could be distinguished easily from the time at which pursuit turned around. The data in Figures 4-6 represent only the initial pursuit and saccade decisions, and Figures 7 and 8 use initial measurements as well as the pursuit turnaround measurements to document pursuit output as a continuous quantity before saccade onset.

\section{Results}

\section{Oculometric results}

We assessed oculomotor responses on the basis of the horizontal direction of pursuit and the vertical direction of the first saccade. Because the two target masks were offset vertically from each other and moved horizontally in opposed directions, responses on each trial consisted of a horizontal pursuit movement and a primarily vertical saccade. Pursuit and saccades almost always eventually tracked the same target, but the initial responses often differed, resulting in four possible combinations of oculomotor responses. Sample traces from four trials illustrating these combinations are shown in Figure 3. These four trials were obtained during a single stimulus condition, in which the target probe ( $60 \%$ contrast) was located below the fixation cross and was visible for $100 \mathrm{msec}$ before being replaced by a leftward-moving 

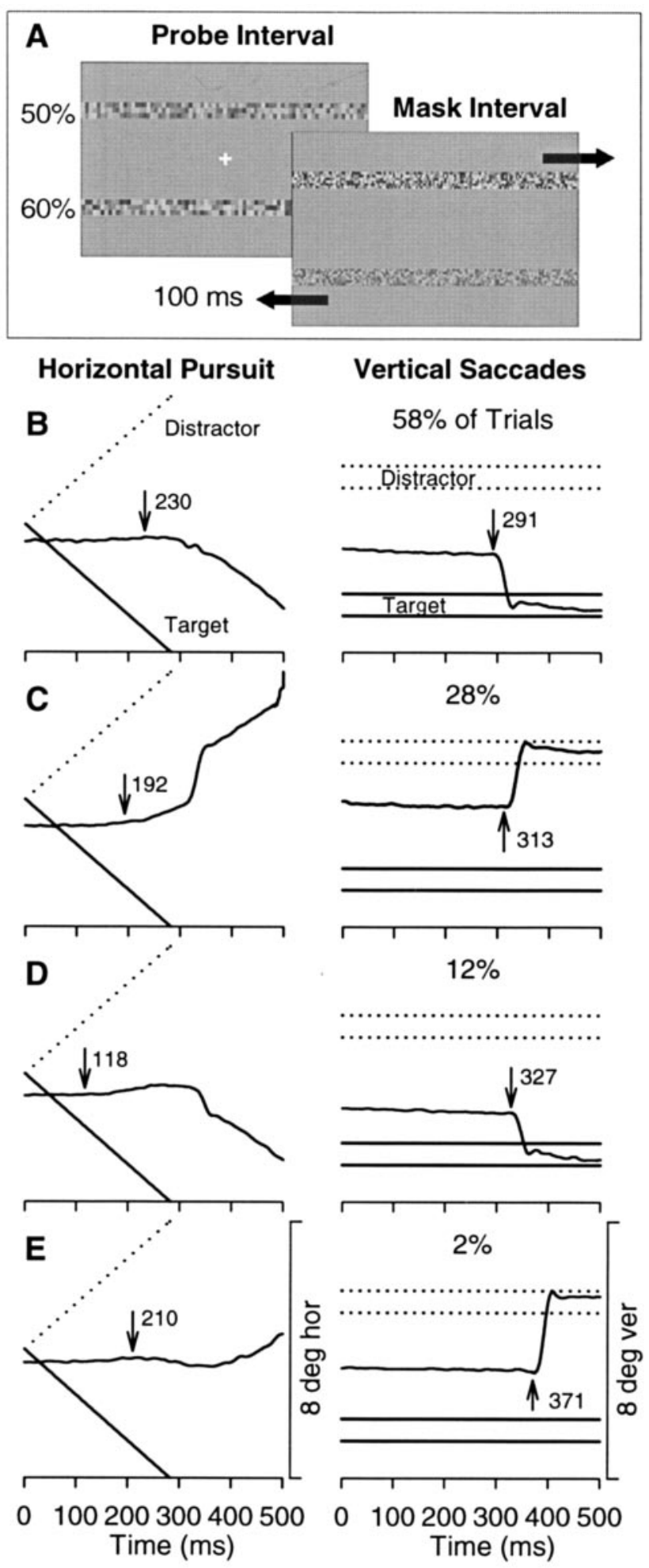

Figure 3. Sample eye position traces collected from subject $D$ on one experimental condition. $A$, During the probe internal, a $60 \%$ contrast probe strip was presented in the lower position for a duration of $100 \mathrm{msec}$. The mask that replaced the target probe was moving to the left. $B-E$ show vertical and horizontal eye position traces for the four possible combinations of correct and incorrect initial decisions for pursuit and saccades. The latency of the initial response is marked with an arrow. In the left column, vertical eye position traces are superimposed on lines delimiting the spatial extent of the target mask (solid) and the distractor mask (broken). The right column shows horizontal eye position traces superimposed on two lines representing the centers of the target mask (solid) and the distractor mask (broken). Because of the large $\left(40^{\circ}\right)$ horizontal extent of the moving masks, any pursuit trajectory parallel to either of the lines representing the centers would be an appropriate tracking response. $B$, Both initial decisions by mask (Fig. 3A). The correct pair of oculomotor responses for this condition was leftward pursuit and a downward saccade, which accounted for $58 \%$ of this subject's responses (Fig. $3 B$ ). The incorrect pair of responses, which occurred on $28 \%$ of the trials for this subject, consisted of an initial upward saccade and rightward pursuit (Fig. 3C). We also observed trials in which the pursuit and saccade responses differed, although these occurred less frequently. These trials consisted of an initial pursuit response that began in one direction and then reversed before or during the saccade. For this condition, the subject initially responded with a correct saccade and incorrect pursuit on $12 \%$ of the trials (Fig. $3 D$ ) and with an incorrect saccade and correct pursuit on $2 \%$ of the trials (Fig. 3E). Average latencies (in milliseconds) for pursuit and saccades for each of the five subjects are given in Table 1.

From these initial eye-movement responses, we constructed pursuit and saccade oculometric functions (Kowler and McKee, 1987; Beutter and Stone, 1998) for both of the relevant parameters of the target: its direction of motion and spatial location. We measured oculomotor performance separately in terms of direction of motion (rightward vs leftward) and location (upward vs downward) because subjects often exhibited direction-ofmotion or location biases, or both, in their responses, especially for stimulus conditions involving short exposure durations. Measuring both types of oculometric functions (motion and location) for pursuit and saccades allowed us to measure two types of bias: bias in one of the directions of horizontal motion and bias to a vertical spatial location. Collapsing across a stimulus parameter to which a subject displays a bias would have represented this bias as lower precision and precluded our ability to detect the bias (Terman and Terman, 1972).

We constructed oculometric functions by plotting the proportions of pursuit and saccade responses as a function of contrast difference. We then fit these data with cumulative Gaussian functions and rejected fits that gave an unacceptable $\chi^{2}$ value $(p<0.05)$. We defined the two fitted parameters of these functions, SD and mean, as the oculometric threshold and oculometric bias, respectively. Thresholds measure the precision with which the subject can identify the target probe and initiate an eye movement to the target mask. Bias measures the tendency of the subject to favor one response over the other. Thus, lower thresholds were associated with steeper oculometric functions, and unbiased responses were associated with oculometric means near zero. To construct the motion oculometric functions, we plotted the proportion of rightward pursuit responses and the proportion of saccade responses to the rightward-moving mask as a function of probe-contrast difference (probe contrast at location of rightward-moving mask minus probe contrast at location of leftward-moving mask). A bias for rightward motion would therefore appear as a leftward shift of the motion oculometric function (i.e., negative values for the mean). For the location oculometric functions, we plotted the proportion of pursuit responses to the upper mask and the proportion of upward saccade responses as a function of probe-contrast difference (contrast of upper probe minus contrast of lower probe). Thus, a bias for the upper spatial location would appear as a leftward shift of the location oculometric function.

$\leftarrow$

pursuit and saccades are correct. C, Both initial decisions are incorrect. D, Pursuit initially follows the distractor mask and then turns around to follow the target mask around the time of the saccade. $E$, Pursuit initially follows the target mask and then later reverses direction to follow the distractor mask. Time 0 in all plots corresponds to mask onset. 
Table 1.

\begin{tabular}{|c|c|c|c|c|c|c|c|c|c|c|c|c|}
\hline \multirow[b]{2}{*}{ Observer } & \multicolumn{6}{|c|}{ Pursuit - exposure duration } & \multicolumn{6}{|c|}{ Saccades - exposure duration } \\
\hline & $50 \mathrm{msec}$ & $100 \mathrm{msec}$ & $150 \mathrm{msec}$ & $200 \mathrm{msec}$ & $300 \mathrm{msec}$ & $500 \mathrm{msec}$ & $50 \mathrm{msec}$ & $100 \mathrm{msec}$ & $150 \mathrm{msec}$ & $200 \mathrm{msec}$ & $300 \mathrm{msec}$ & $500 \mathrm{msec}$ \\
\hline B & 185 & 174 & 166 & 165 & 165 & 156 & 290 & 264 & 244 & 242 & 239 & 221 \\
\hline$S$ & 175 & 167 & 168 & 171 & 167 & 157 & 220 & 200 & 201 & 208 & 206 & 186 \\
\hline $\mathrm{N}$ & 165 & 149 & 140 & 138 & 133 & 128 & 271 & 239 & 215 & 210 & 203 & 200 \\
\hline $\mathrm{R}$ & 171 & 160 & 152 & 149 & 147 & 137 & 261 & 228 & 213 & 213 & 209 & 193 \\
\hline D & 204 & 190 & 184 & 183 & 185 & 174 & 335 & 298 & 270 & 267 & 262 & 243 \\
\hline
\end{tabular}

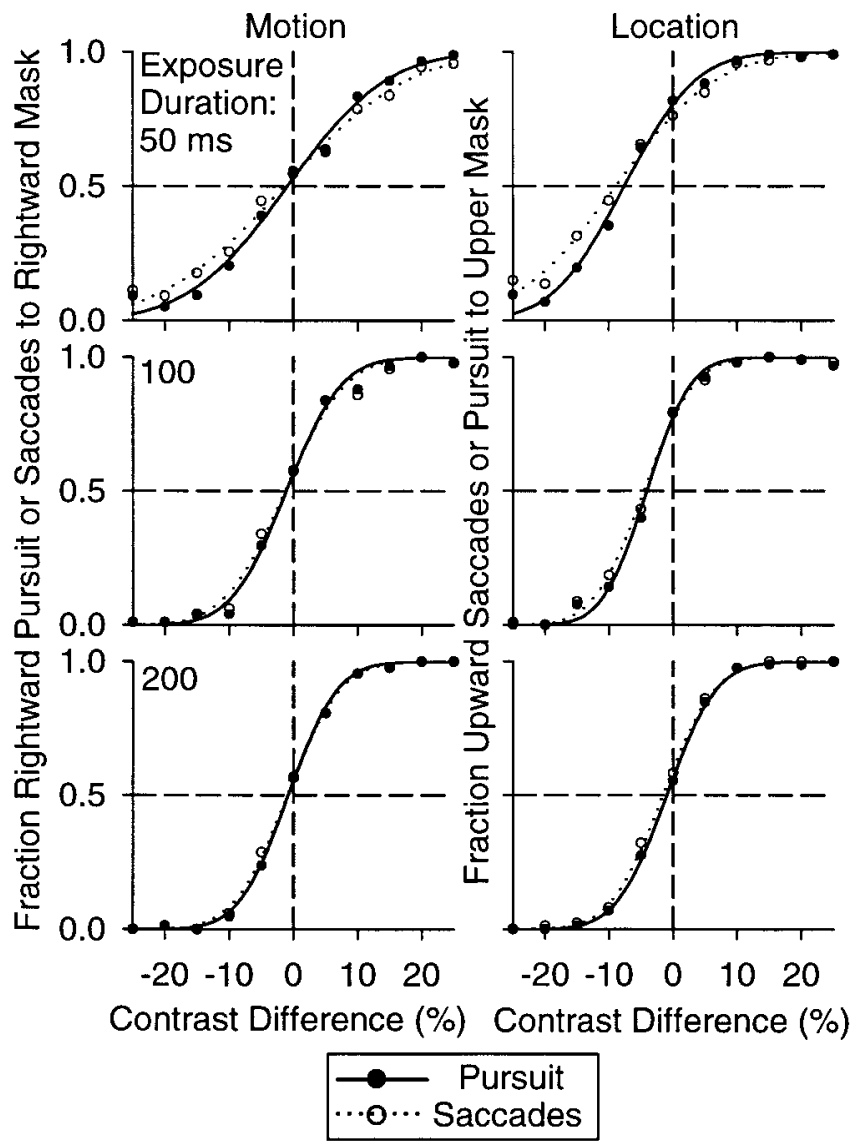

Figure 4. Oculometric functions for pursuit and saccades for three exposure durations. The left column shows oculometric functions from the motion set of measurements. Each data point represents the fraction of rightward pursuit (filled symbols) and saccades to the rightward mask (unfilled symbols) plotted as a function of probe contrast difference. The right column shows oculometric functions from the location set of measurements, in which each data point represents the fraction of pursuit choices to the upper mask (filled symbols) and the fraction of upward saccade choices (unfilled symbols) plotted as a function of probe contrast difference. The smooth functions are cumulative normal fits to the data. Each row of graphs shows data from one exposure duration.

In Figure 4 we show the motion and location sets of oculometric functions for naive subject N. For the purpose of this figure, we pooled the data across the 10 experimental sessions to construct these functions. Each panel contains one set of pursuit and saccade oculometric functions for one exposure duration. The left column of "Motion" oculometric functions shows the proportion of rightward pursuit responses (filled circles) and the proportion of saccade responses (unfilled circles) to the rightward moving mask, both measured independently of the vertical target location. Each data point in these functions is based on the outcome of $\sim 100$ trials. The complementary right column of "Location" oculometric functions shows the proportion of up- ward saccade responses and the proportion of pursuit responses to the upper mask, both measured independently of the direction of motion of the target mask. The curves superimposed on the data show the best cumulative Gaussians fits to the pursuit (solid line) and saccade (dotted line) oculometric functions.

Exposure duration had a large effect on the precision (i.e., threshold) and accuracy (i.e., bias) of both pursuit and saccades. When subject $\mathrm{N}$ had only $50 \mathrm{msec}$ to view the probe patches, her pursuit and saccade oculometric functions had shallow slopes for both motion and location measurements. Accordingly, her oculometric thresholds were high (motion, $12.2 \%$ for pursuit and $15.3 \%$ for saccades; location, $8.9 \%$ for pursuit and $12.4 \%$ for saccades). The location oculometric functions showed a strong bias favoring upward saccades and pursuit to the upper mask ( $-7.6 \%$ for pursuit and $-8.9 \%$ for saccades). These bias values signify that the lower probe had to be 7.6 and $8.9 \%$ greater in contrast for this subject to pursue or make a saccade to the lower mask on half of the trials, respectively. This subject did not display a bias for motion, as indicated by the fact that her oculometric functions had mean values near zero. When she had $200 \mathrm{msec}$ to view the probe patches, both her motion and location thresholds were lower (motion, $6.1 \%$ for pursuit and 6.5\% for saccades; location, $6.1 \%$ for pursuit and $6.4 \%$ for saccades) and her location bias was smaller $(-0.98 \%$ for pursuit and $-1.6 \%$ for saccades).

We observed similar effects of exposure duration on oculometric thresholds for all subjects. To test the significance of this effect, we fit the pursuit and saccade oculometric functions from each subject for each of the 10 experimental sessions individually. The cumulative Gaussian generally provided a good fit to our data; the fits were rejected for only 22 of the 1200 oculometric functions in our data set $\left(\chi^{2} ; p<0.05 ; 5\right.$ subjects $\times 6$ exposure durations $\times 10$ sessions $\times 2$ eye movements $\times 2$ sets of measurements). We plotted pursuit and saccade thresholds (Fig. 5, circles) and biases (triangles) as a function of exposure duration. Every subject showed a significant effect of exposure duration on pursuit and saccade thresholds ( $p<0.01$; two-way ANOVA).

Pursuit and saccade thresholds tended to be the same, even as they both decreased with exposure duration. On the basis of a two-way ANOVA, four of the five subjects did not show a significant difference between pursuit and saccade motion thresholds, although one subject $(\mathrm{R})$ did show a significant difference, primarily at longer exposure durations. The thresholds taken from the location sets of oculometric functions showed significant differences between pursuit and saccade thresholds for two subjects (subjects $\mathrm{S}$ and R; $p<0.05$; two-way ANOVA).

All subjects showed an effect of exposure duration on motion or location bias, or both (Fig. 5). Pursuit and saccade biases for a direction of motion were displayed by two of five subjects, subject $\mathrm{R}$ favoring leftward motion (triangles above zero) and subject $\mathrm{D}$ favoring rightward motion (triangles below zero). These subjects showed a significant effect of exposure duration on motion biases 


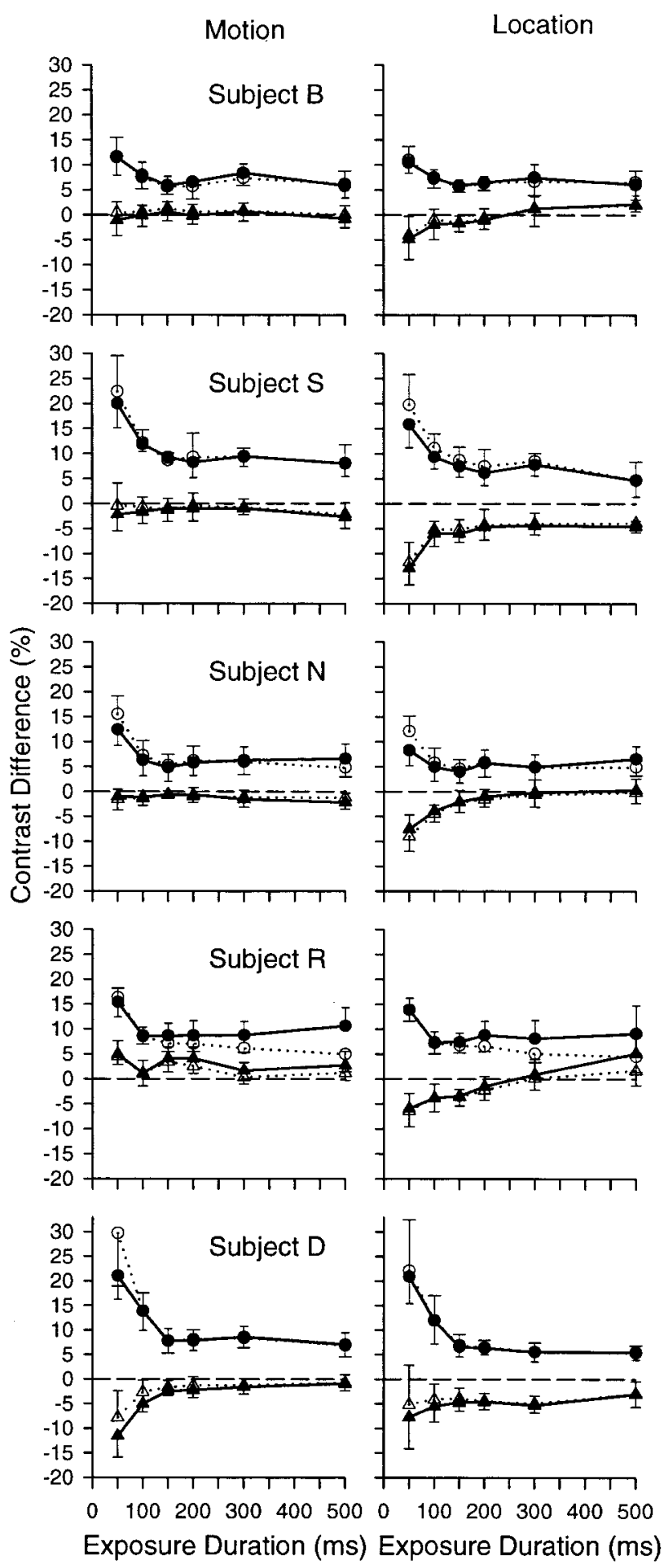

Figure 5. Oculometric thresholds and biases for the five experimental subjects. The left column shows location thresholds (circles) and biases (triangles) for pursuit (solid lines and filled symbols) and saccades (broken lines and unfilled symbols) plotted as a function of exposure duration. The right column shows the corresponding set of motion thresholds and biases. Error bars represent the SD across the 10 experimental sessions. For clarity, each error bar is unidirectional. Each row of graphs shows data from one subject. for both oculomotor systems ( $p<0.05$; two-way ANOVA). All subjects except subject $\mathrm{D}$ had significant upward location biases that became less severe as exposure duration increased ( $p<0.05$; two-way ANOVA).

Pursuit and saccade biases tended to be similar. For the two subjects with motion biases (subjects $\mathrm{R}$ and $\mathrm{D}$ ), pursuit and saccade biases followed a similar course as exposure duration increased. The same qualitative point is true of the upward location biases as well; the amplitude of pursuit and saccade biases decrease similarly as exposure duration increases. No subject showed a significant difference between pursuit and saccade biases for either motion or location ( $p<0.05$; two-way ANOVA).

\section{Trial-by-trial results}

Although the oculometric analysis showed that pursuit and saccade thresholds tended to be the same on average, it cannot test whether both systems behaved similarly on individual trials. To compare pursuit and saccade responses on a trial-by-trial basis, we therefore measured the fraction of trials on which pursuit and saccades initially followed the same stimulus (i.e., both responses were either "correct" or "incorrect") for each stimulus condition. Because these fractions were similar across the five subjects, we pooled data across subjects to produce the observed fractions of "same" pursuit and saccade responses. We compared the observed fraction of same decisions (Fig. 6, solid line) with the fraction expected from completely dependent (dotted line) and completely independent mechanisms (dashed line). The predicted fraction of same decisions from completely dependent mechanisms would be unity, because the two systems would always concur. The fractions associated with independent outcomes were calculated from the equation: $F_{\text {independent }}=F_{\text {pursuit }}$ * $F_{\text {saccades }}+\left(1-F_{\text {pursuit }}\right) *\left(1-F_{\text {saccades }}\right)$, in which $F_{\text {pursuit }}$ and $F_{\text {saccades }}$ are the fraction of correct pursuit and saccade trials for any given stimulus condition, respectively. Each panel in Figure 6 shows the observed frequency of same pursuit and saccade responses and the predictions from dependent and independent mechanisms plotted as a function of contrast difference (upper probe contrast minus lower probe contrast) for a single exposure duration (50-200 msec). "Sameness" at the longest exposure durations ( 300 and $500 \mathrm{msec}$ ) was similar to the values at $200 \mathrm{msec}$. The separation between the solid and dashed curves in Figure 6 indicates that the observed fraction of same responses was consistently higher than that expected if the two responses were independent. As indicated by the filled symbols, this difference was significant for the majority of the stimulus conditions $(p<0.05$; Wilcoxon rank sum test). For every exposure duration, we found that the observed fraction of same responses was always significantly different from $1.0\left(\chi^{2} ; p<0.0001\right)$. Thus, our data did not match the predictions based on mechanisms that were completely dependent or completely independent.

\section{Continuous pursuit choice results}

The trial-by-trial analysis showed a significant number of disagreements between pursuit and saccades, which reflect an initial pursuit decision that differs from the later saccade. On these trials, pursuit reversed some time before or around the time of the saccade to bring the systems into agreement. To quantify the time course of pursuit turnarounds, we measured the sameness of pursuit and saccade choices continuously around the time of the saccade. We selected a condition with a large proportion of pursuit and saccade disagreements (50 msec exposure duration; 0\% contrast difference) and compared the observed fraction of same decisions at every millisecond in the interval from $200 \mathrm{msec}$ be- 


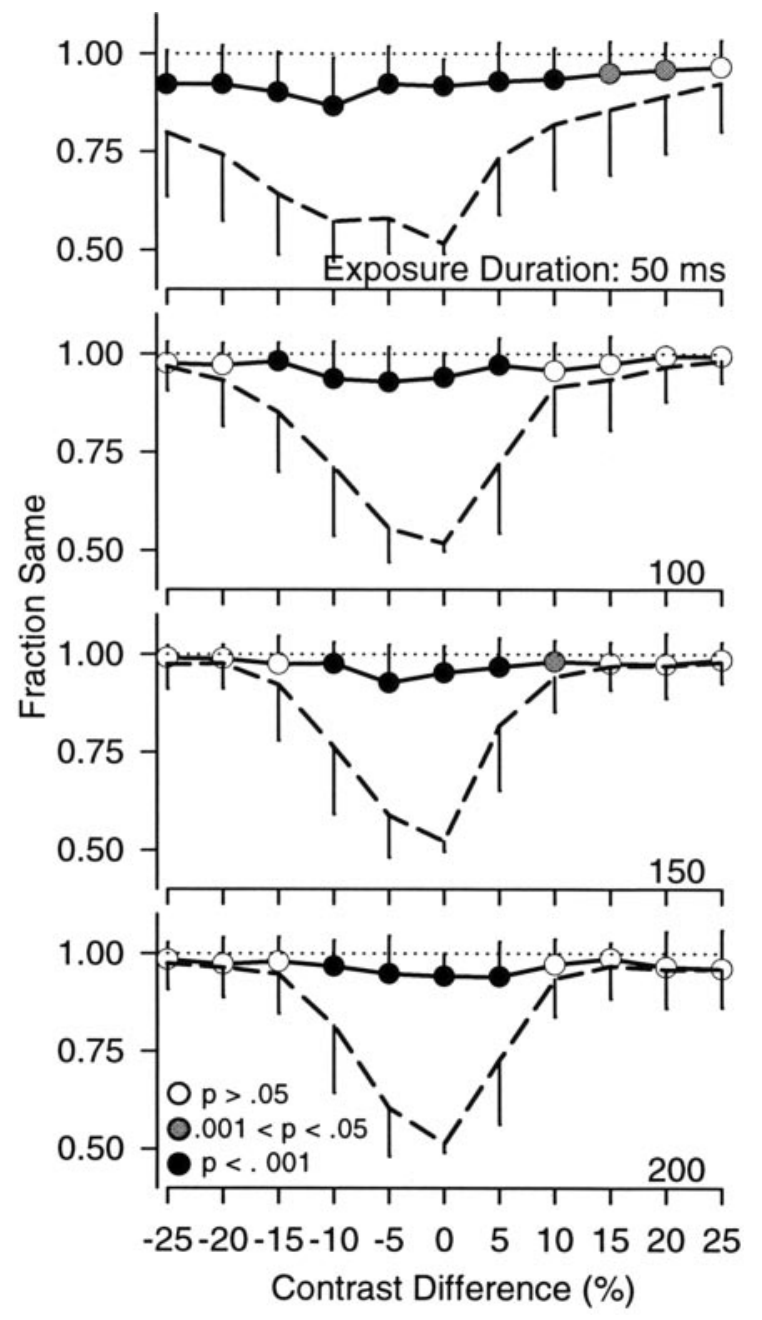

Figure 6. Analysis of sameness of pursuit and saccade decisions on a trial by trial basis. The circles and solid lines in each graph plot the fraction of trials on which initial pursuit and saccade decisions agreed, plotted as a function of contrast difference. The two lines plot the proportion of trials in which the two decisions would be expected to be the same if the decision-making mechanisms were independent (broken) or completely dependent (dotted). Filled symbols indicate those cases in which the observed fraction of same decisions was significantly greater than predicted by independent mechanisms. The error bars represent the SD across the 10 experimental sessions and the six observers. Each set of axes shows data from one exposure duration.

fore to $50 \mathrm{msec}$ after the saccade. For example, if pursuit initiated in one direction $100 \mathrm{msec}$ before the saccade and was brought into concordance $50 \mathrm{msec}$ before the saccade, we would count every millisecond from -100 to -51 as a disagreement and every millisecond from -50 to +50 as an agreement. Of the 868 trials included in this analysis, we found only one trial on which the pursuit and saccadic systems were not in agreement by $50 \mathrm{msec}$ after the saccade. We measured the time course of pursuit turnarounds on every session and compared this time course across the 10 experimental sessions for each subject. We included time points at which we observed five or more pursuit responses in at least two sessions.

Pursuit and saccades disagree most long before the saccade. Figure 7 shows the time course of pursuit turnarounds for each of our five experimental subjects. Each panel in Figure 7 shows one subject's observed frequency of same pursuit and saccade responses, plotted as a function of time with respect to the saccade. The continuous line shows the mean across sessions, and the

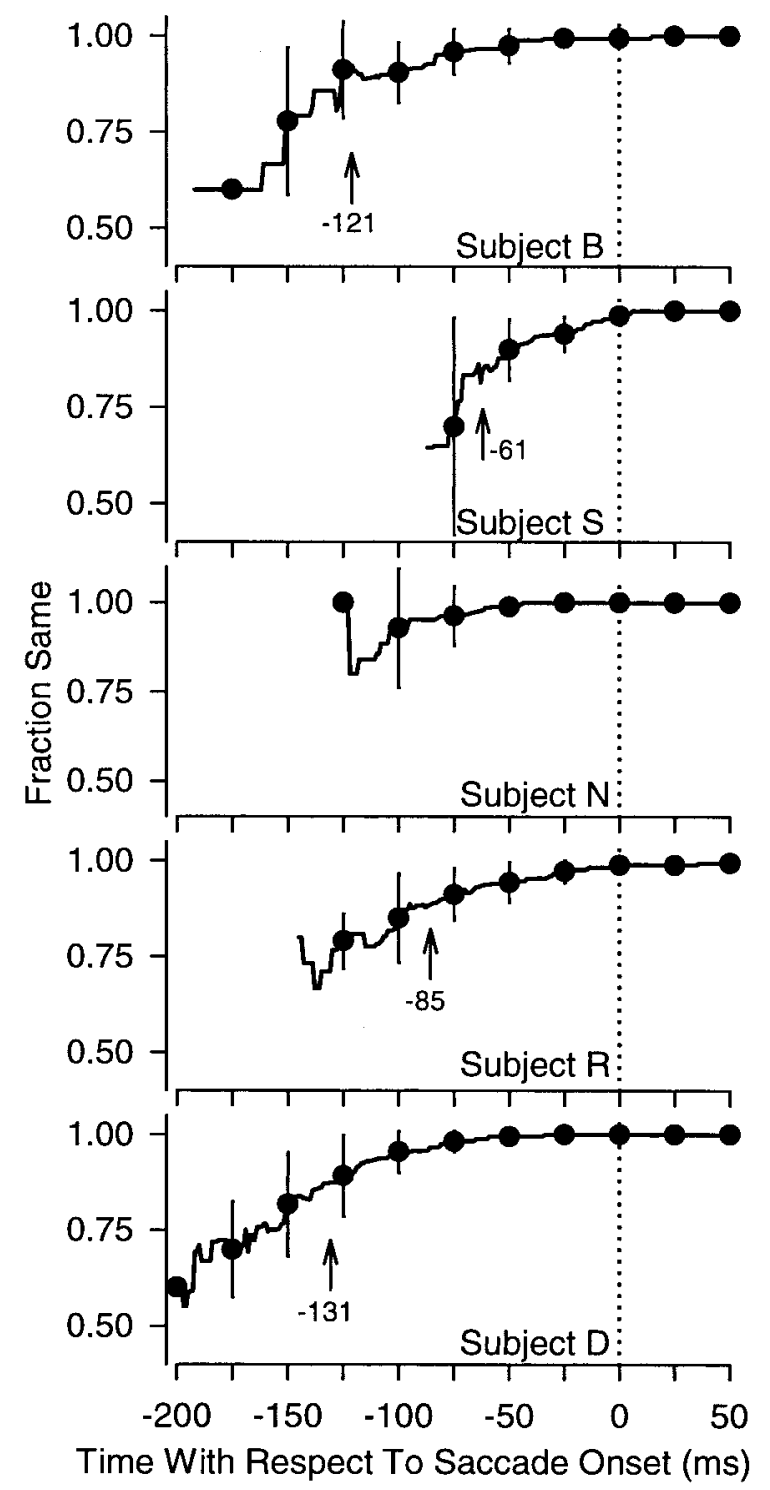

Figure 7. Perisaccadic analysis of sameness of pursuit and saccade decisions. The solid line in each graph plots the proportion of trials on which pursuit predicts the saccadic choice, plotted as a function of time with respect to saccade onset. The solid line is an average across the 10 time courses measured for each of the experimental sessions. For clarity, the variance in these measurements is shown at 25 msec intervals, represented by the filled circles (mean) with error bars ( $\pm 1 S D)$. Each set of axes shows data from one experimental subject.

error bars plotted at $25 \mathrm{msec}$ intervals represent the SD. Four of the five subjects showed a significant increase in pursuit and saccade sameness in the $200 \mathrm{msec}$ interval before the saccade (oneway ANOVA; $p<0.05$ ). To quantify the timing of this effect, we performed a post hoc comparison across our measurement interval for the four subjects whose time course data showed significant differences. We used the fraction of same responses at the time of the saccade (mean $>0.99$ for every subject) as a control group and compared the sameness at this time point with every other time point in the measurement interval (Dunnet multiple comparisons; $p<0.05$ ). We found that by at least $61 \mathrm{msec}$ before the saccade, the proportion of trials on which pursuit and saccades disagreed was no longer significantly different from the proportion of trials on which these systems disagreed at saccade onset, for all subjects. The point in time at which the difference 
between pursuit and saccades was no longer significant is marked with an arrow in the graph for each subject.

Although the time course of pursuit turnarounds shows that the two systems disagree most long before the saccade, it cannot address what factors might be contributing to disagreements between pursuit and saccades. To investigate whether a timevarying motor bias could be responsible for pursuit and saccade disagreements, we repeated the oculometric analysis at various points in time with respect to the saccade, in the spirit of the previous analysis. We constructed both motion and location oculometric functions (Fig. 4) at every millisecond in the interval from $200 \mathrm{msec}$ before to $50 \mathrm{msec}$ after the saccade. Again, we used data only from the $50 \mathrm{msec}$ exposure duration, and the assignment of pursuit responses to the 251 time bins around saccade onset was performed as described previously. We collapsed data across sessions to allow for the greatest possible number of observations and fit each of the 251 oculometric functions for each subject with a cumulative Gaussian. The cumulative Gaussian provided a good fit to these data out to at least $75 \mathrm{msec}$ before the saccade. We included time points with an acceptable $\chi^{2}$ value $\left(\chi^{2} ; p \geq 0.05\right)$ and at least 10 observations for each condition. To quantify whether any change over time was significant, we performed this analysis again using data from individual sessions. Because the number of observations in each oculometric function was approximately decimated, we were able to obtain reliable measurements over a much smaller portion of the interval.

Pursuit bias for a direction of motion did not change significantly for any subject before the saccade. The left column in Figure 8 shows the time course of pursuit motion bias (unfilled circles) and location bias (filled circles) around the time of the saccade for each of our experimental subjects. The right column in Figure 8 shows the corresponding pursuit threshold measurements. Although subject D's pursuit showed a bias for rightward motion when collapsed across sessions (left column, fourth panel, gray trace), we did not find significant changes in pursuit motion biases or thresholds using repeated measures (one-way ANOVA; $p<0.05$ ).

Although most subjects showed a significant bias for the upper spatial location in the oculometric analysis of initial decisions, pursuit bias for only one subject varied over time before the saccade. When collapsed across sessions, subjects $\mathrm{B}$ and $\mathrm{N}$ showed slight changes in pursuit bias for the upper spatial location over time, and subjects $\mathrm{R}$ and $\mathrm{D}$ showed more dramatic changes. We found that only one subject (R) showed a significant change for pursuit bias in the interval over which we could make repeated measures (one-way ANOVA; $p<0.05$ ). No subject showed a significant change for thresholds. We performed a post hoc comparison similar to the one described above across our measurement interval for subject $\mathrm{R}$, whose bias data showed a significant difference. We used the measurements of pursuit location bias at the time of the saccade as a control group, with which we compared pursuit bias at every other time point in the measurement interval. The difference between pursuit and saccade bias was no longer significant at $75 \mathrm{msec}$ before the saccade (Fig. 8, arrow) (Dunnet multiple comparisons; $p<0.05$ )

\section{Discussion}

We used a contrast discrimination task, and techniques from psychophysics, to compare the performance of the pursuit and saccadic systems. We found that pursuit and saccades exhibited similar thresholds and biases that decreased together as the period of visual processing available for discrimination was extended. Comparing choices on individual trials, we found that
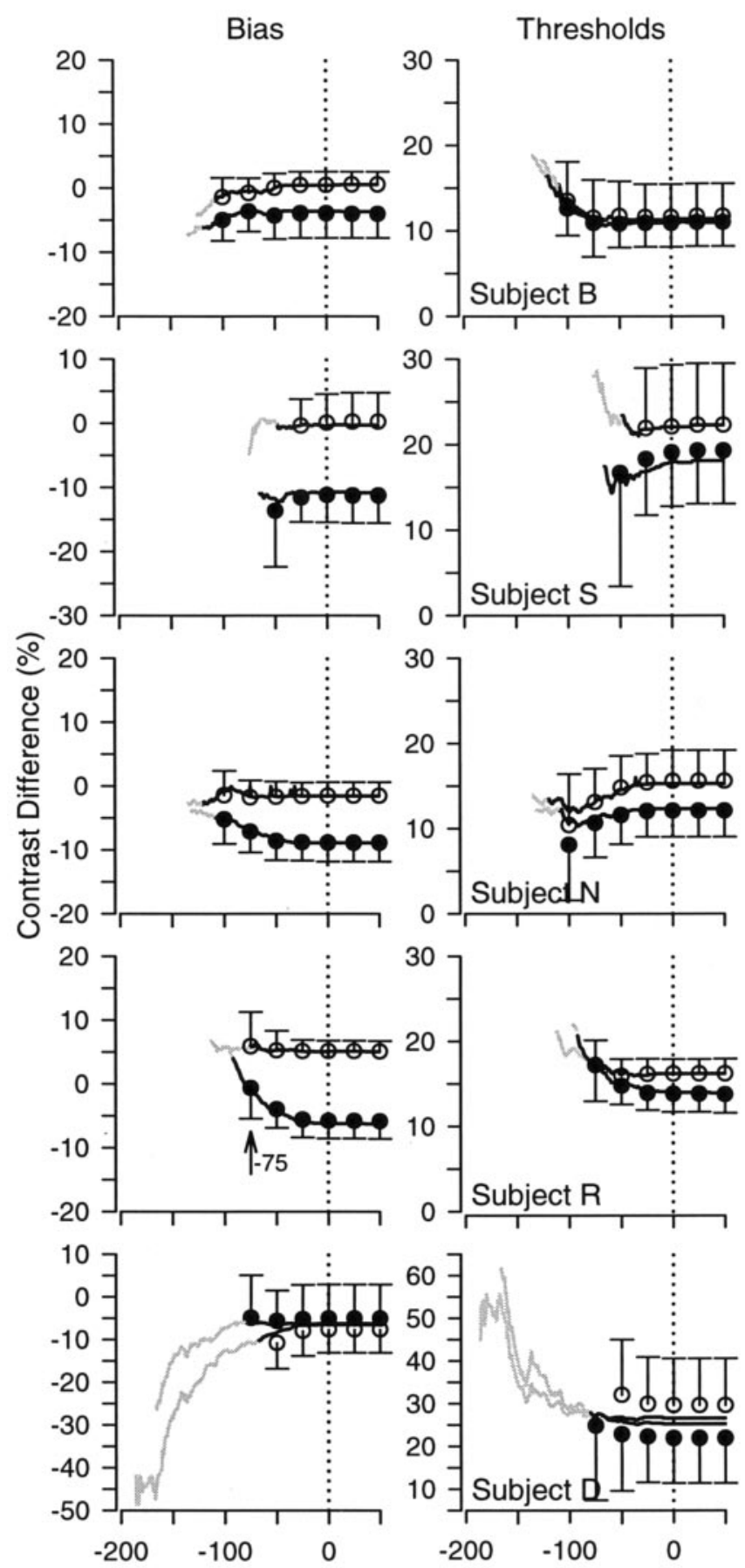

Time With Respect To Saccade Onset (ms)

Figure 8. Perisaccadic oculometric thresholds and biases. The left column shows pursuit motion bias (open circles) and location bias (filled circles) plotted as a function of time with respect to saccade onset. The right column shows the corresponding set of thresholds measurements. The solid line in each graph plots measurements using data collapsed across all 10 experimental sessions. The circles with error bars show the mean and SD of repeated measures made across individual sessions, plotted at $25 \mathrm{msec}$ intervals. For clarity, each error bar is unidirectional. In some cases, the mean of the repeated measurements differs from the value obtained when the data were collapsed across session. The gray portion of the solid line represents the temporal region where repeated measures across sessions were not possible. Subject R showed a time-varying location bias for pursuit, which deviates significantly from pursuit bias at saccade onset in the interval from 92 to $76 \mathrm{msec}$ before the saccade. The time point at which this difference is no longer significant is marked with an arrow. Pursuit thresholds and motion bias did not change significantly over time for any subject. 
the initial pursuit response differed from the subsequent saccade on up to $13 \%$ of the trials. Exploiting the continuous output provided by pursuit, we investigated the time course of these choices on a millisecond by millisecond basis before saccade onset. On trials when pursuit initially disagreed with the subsequent saccade, pursuit quickly reversed direction to predict the saccade choice that followed. Examining the time course of pursuit thresholds and bias, we found that pursuit choices tended to display spatial biases that changed over time, eventually matching the saccade bias. Together, these results support the idea that the programming of pursuit and saccades involves shared response preparation.

\section{Comparison with previous studies}

At least superficially, our study involved stimuli and methods that were quite different from those used previously. First, rather than using small discrete targets, we used long horizontal strips. Other studies have used extended moving stimuli for pursuit to reduce or eliminate catch-up saccades (Watamaniuk and Heinen, 1999; Krauzlis and Adler, 2001); in our case, the horizontal configuration maintained the stimuli at a constant eccentricity so that the amplitude of the required saccade did not change with pursuit latency. The vertical offset of the strips $\left(2^{\circ}\right)$ was chosen to be large enough to elicit saccades reliably yet small enough for the horizontal motion to drive presaccadic pursuit, allowing us to record both a pursuit and saccadic movement on each trial. Previous studies have examined pursuit and saccades in separate types of trials (Krauzlis and Miles, 1996; Krauzlis et al., 1999), usually with the motion and location of the pursuit stimulus carefully adjusted to minimize the intrusion of catch-up saccades (Rashbass, 1961). By evoking both pursuit and a saccade on each trial, however, we could exclude the possibility of subjects adopting different strategies on pursuit versus saccade trials and could also compare the performance of the two systems on a trial by trial basis.

Second, our stimuli were presented in two distinct stages: briefly presented stationary probe stimuli necessary for the contrast discrimination followed by moving masks that elicited eye movement responses. Although the two-stage stimulus is unusual, its effect is similar to more typical pursuit choice experiments. For example, previous studies of pursuit target selection have used color as a cue (Ferrera and Lisberger, 1995; Krauzlis et al., 1999); subjects must first discriminate the color of the stimuli and then track the motion of the indicated stimulus. If the discrimination is made more difficult, subjects can partially compensate by delaying their responses. In contrast, our two-stage stimulus explicitly separates the discrimination and response steps, thereby disassociating the difficulty of the discrimination from reaction time.

\section{Possible mechanisms underlying the coordination of pursuit and saccades}

We have found that initial pursuit and saccade choices are usually, but not always, the same. At what stage in the cascade of neural events between visual sensation and motor action does this coordination arise? A simplified functional description of this complicated cascade includes three components: visual processing, response preparation, and motor execution. Visual processing involves the encoding of the visual stimulus, first in the retina and continuing through striate and extrastriate cortex. Both pursuit and saccades depend on V1 (Zee et al., 1987) and are influenced by later visual processing areas (Newsome et al., 1985; Komatsu and Wurtz, 1989). Response preparation acts on the result of visual processing by modifying the visual representation according to the behavioral relevance of each object (Treisman and Gelade, 1980; Findlay and Walker, 1999; Moore and Fallah, 2001; Cutrell and Marrocco, 2002; Bisley and Goldberg, 2003; Moore and Armstrong, 2003). Finally, execution of the movement requires activity in output pathways, generating the final motor commands.

The visual processing stage can explain some of our results. Pursuit and saccade choices both rely on the early processing of visual information (e.g., retina, V1) to perform the contrast discrimination. Because the variability introduced at early visual stages by our "noisy" visual stimuli was shared by the two systems, one might expect a strong correlation between the choices even if they were determined by different downstream mechanisms. This does not imply that both choices were completed at early visual stages; the shared input signal could exert its effect anywhere downstream as it propagated through the rest of the system. In fact, because the stimuli used for the contrast discrimination were replaced by masking stimuli well before movement onset, it is unlikely that the choice occurred at early visual stages. By the time of eye movement initiation, the sensory representation of the probe strips had been masked for between 180 and 275 msec (average pursuit and saccade latencies, $50 \mathrm{msec}$ condition), making it doubtful that early visual stages could have maintained any representation of the probe stimuli. Thus, the experimental design points to coordinated eye movement choice at a site downstream of early visual processing.

Can the visual processing stage account for disagreements between pursuit and saccades? Pursuit depends heavily on visual motion processing accomplished in the medial temporal (MT) and medial superior temporal (MST) areas of extrastriate cortex (Newsome et al., 1985; Dürsteler and Wurtz, 1988), whereas these areas are not critical for saccade production. One possibility is that different roles played by these areas are responsible for different choices made by the two systems. However, neurons in MT and MST exhibit only a weak correlation with pursuit choice; their activity is determined primarily by the visual stimulus (Ferrera and Lisberger, 1997; Recanzone and Wurtz, 2000). Thus, like neurons earlier in the visual pathways, MT and MST neurons would have primarily represented information about the mask stimuli rather than the probe stimuli or eye movement choices.

The similarity in pursuit and saccade response biases points to coordination at the level of response preparation. Although in principle these biases could have been caused by either unequal weighting of sensory signals (Green and Swets, 1988) or asymmetries in downstream decision making (Link and Heath, 1975; Green and Swets, 1988), several aspects of our data argue that they were not sensory in origin. First, we measured response biases at movement initiation, after the informative probe stimuli had been masked. Second, if unequal weighting of sensory inputs had caused the biases, they should have increased with extended viewing of the probe stimuli and not decreased, as we observed (Fig. 5). Third, the changes in pursuit bias before saccade onset (Fig. 8) implicate a bias at the stage of response preparation. Pursuit responses exhibited time-varying biases, especially for spatial location, that stabilized before the saccade. The time course of these biases is consistent with a downstream locus because response preparation continued throughout the mask interval, unlike visual processing, which was truncated by the mask onset. The gradual changes that we found in threshold (Fig. 8, right column), bias (Fig. 8, left column), and trial by trial concordance (Fig. 7) support the idea that the pursuit response is gov- 
erned by a time-varying preparatory signal that also determines the saccade choice.

Previous studies have provided seemingly different views on how and whether pursuit target selection and saccade target selection are coordinated. One view suggests that pursuit target selection is serially linked to saccade execution (Gardner and Lisberger, 2001, 2002), whereas another suggests that pursuit and saccade target selection use the same neurons but different decision criteria (Krauzlis and Dill, 2002). Our current results show that the output of the pursuit system can predict the saccadic choice at least $60 \mathrm{msec}$ before saccade onset. Similarly, other studies have shown presaccadic pursuit to be at least weakly selective before saccade onset (Gardner and Lisberger, 2001, their Fig. 5e). These observations indicate that pursuit target selection need not be strictly time locked to saccade occurrence. Instead, saccadic target-selection signals, as they develop over time, may regulate the strength of the visual motion inputs that drive pursuit. In our experiment, the location of the target mask was predictable, although its motion was not. The target-selection signal for saccades, specifying the location of the target mask as the saccade endpoint, may often have been well developed before mask onset. In this case, at mask onset, only the motion signal at the preselected saccade endpoint would drive the pursuit system. The complete agreement between pursuit and saccades on easy discriminations at long exposure durations (Fig. 5) supports this idea.

Our findings argue that the signals guiding pursuit target selection are the same as those involved in the covert preparation of saccades. This result corroborates the recent observation that the preparatory activity of saccade-related neurons in the superior colliculus can predict pursuit choices (Krauzlis and Dill, 2002). Spatially organized motor maps may not be the most obvious vehicles for selecting targets for pursuit, but this idea is consistent with the observation that both systems showed the same bias for spatial location (Figs. 5, 8) and the previous finding that previous information about stimulus location has larger effects on pursuit latency than previous information about stimulus motion (Adler et al., 2002). By defining the response goal for pursuit and saccades within a common spatial framework, higher-order factors-attention, perception, and cognition-could influence both systems in parallel.

\section{References}

Adler SA, Bala J, Krauzlis RJ (2002) Primacy of spatial information in guiding target selection for pursuit and saccades. J Vision 2:627-644.

Beutter BR, Stone LS (1998) Human motion perception and smooth eye movements show similar directional biases for elongated apertures. Vision Res 38:1273-1286.

Bisley JW, Goldberg ME (2003) Neuronal activity in the lateral intraparietal area and spatial attention. Science 299:81-86.

Cutrell EB, Marrocco RT (2002) Electrical microstimulation of primate posterior parietal cortex initiates orienting and alerting components of covert attention. Exp Brain Res 144:103-113.

DeValois RL, DeValois KK (1991) Spatial vision. New York: Oxford UP.

Dürsteler MR, Wurtz RH (1988) Pursuit and optokinetic deficits following chemical lesions of cortical areas MT and MST. J Neurophysiol 60:940-965.

Eckstein MP, Beutter BR, Stone LS (2001) Quantifying the performance limits of human saccadic targeting during visual search. Perception 30:1389-1401.

Ferrera VP, Lisberger SG (1995) Attention and target selection for smooth pursuit eye movements. J Neurosci 15:7472-7484.

Ferrera VP, Lisberger SG (1997) Neuronal responses in visual areas MT and MST during smooth pursuit target selection. J Neurophysiol 78:1433-1446.

Findlay JM, Walker R (1999) A model of saccade generation based on parallel processing and competitive inhibition. Behav Brain Sci 22:661-674.

Fischer B, Boch R (1983) Saccadic eye movements after extremely short reaction times in the monkey. Brain Res 260:21-26.
Fischer B, Weber H (1993) Express saccades and visual attention. Behav Brain Sci 16:553-610.

Gardner JL, Lisberger SG (2001) Linked target selection for saccadic and smooth pursuit eye movements. J Neurosci 21:2075-2084.

Gardner JL, Lisberger SG (2002) Serial linkage of target selection for orienting and tracking eye movements. Nat Neurosci 5:892-899.

Green DM, Swets JA (1988) Signal detection theory and psychophysics. Los Altos, CA: Peninsula.

Knox PC (1996) The effect of the gap paradigm on the latency of human smooth pursuit of eye movement. NeuroReport 7:3027-3030.

Knox PC (2001) The effect of stationary distractors on pursuit initiation: distractors operate as objects. Soc Neurosci Abstr 27:784.8.

Knox PC, Bekkour T (2002) Non-target influences on the initiation of smooth pursuit. Prog Brain Res 140:211-224.

Komatsu H, Wurtz RH (1989) Modulation of pursuit eye movements by stimulation of cortical areas MT and MST. J Neurophysiol 62:31-47.

Kowler E, McKee SP (1987) Sensitivity of smooth eye movement to small differences in target velocity. Vision Res 27:993-1015.

Krauzlis RJ, Adler SA (2001) Effects of directional expectations on motion perception and pursuit eye movements. Vis Neurosci 18:365-376.

Krauzlis R, Dill N (2002) Neural correlates of target choice for pursuit and saccades in the primate superior colliculus. Neuron 35:355-363.

Krauzlis RJ, Miles FA (1996) Release of fixation for pursuit and saccades in humans: evidence for shared inputs acting on different neural substrates. J Neurophysiol 76:2822-2833.

Krauzlis RJ, Stone LS (1999) Tracking with the mind's eye. Trends Neurosci 22:544-550.

Krauzlis RL, Zivotofsky AZ, Miles FA (1999) Target selection for pursuit and saccadic eye movements in humans. J Cognit Neurosci 11:641-649.

Link SW, Heath RA (1975) A sequential theory of psychological discrimination. Psychometrika 40:77-105.

Lisberger SG, Ferrera VP (1997) Vector averaging for smooth pursuit eye movements initiated by two moving targets in monkeys. J Neurosci 17:7490-7502.

Lisberger SG, Morris EJ, Tychsen L (1987) Visual motion processing and sensory-motor integration for smooth pursuit eye movements. Annu Rev Neurosci 10:97-129.

Merrison AF, Carpenter RH (1995) “Express” smooth pursuit. Vision Res 35:1459-1462.

Moore T, Armstrong KM (2003) Selective gating of visual signals by microstimulation of frontal cortex. Nature 421:370-373.

Moore T, Fallah M (2001) Control of eye movements and spatial attention. Proc Natl Acad Sci USA 98:1273-1276.

Newsome WT, Wurtz RH, Dürsteler MR, Mikami A (1985) Deficits in visual motion processing following ibotenic acid lesions of the middle temporal visual area of the macaque monkey. J Neurosci 5:825-840.

Rashbass C (1961) The relationship between saccadic and smooth tracking eye movements. J Physiol (Lond) 159:326-338.

Recanzone GH, Wurtz RH (2000) Effects of attention on MT and MST neuronal activity during pursuit initiation. J Neurophysiol 83:777-790.

Saslow MG (1967) Effects of components of displacement-step stimuli upon latency for saccadic eye movement. J Opt Soc Am 57:1024-1029.

Schall JD, Thompson KG (1999) Neural selection and control of visually guided eye movements. Annu Rev Neurosci 22:241-259.

Sparks DL, Mays LE (1990) Signal transformations required for the generation of saccadic eye movements. Annu Rev Neurosci 13:309-336.

Swift D, Panish S, Hippensteel B (1997) The use of VisionWorks in visual psychophysics research. Spat Vis 10:471-477.

Terman M, Terman JS (1972) Concurrent variation of response bias and sensitivity in an operant-psychophysical test. Percept Psychophys 11:428-432.

Treisman AM, Gelade G (1980) A feature-integration theory of attention. Cognit Psychol 12:97-136.

Walker R, Deubel H, Schneider WX, Findlay JM (1997) Effect of remote distractors on saccade programming: evidence for an extended fixation zone. J Neurophysiol 78:1108-1119.

Watamaniuk SN, Heinen SJ (1999) Human smooth pursuit direction discrimination. Vision Res 39:59-70.

Zee DS, Tusa RJ, Herdman SJ, Butler PH, Güçer G (1987) Effects of occipital lobectomy upon eye movements in primate. J Neurophysiol 58:883-907. 